\title{
Fluorescence Confocal Microscopy Imaging Denoising with Photobleaching
}

\author{
Isabel Rodrigues $^{1,2}$, João Xavier ${ }^{2,3}$ and João Sanches ${ }^{2,3}$
}

\begin{abstract}
The Fluorescence Confocal Microscopy (FCM) is nowadays one of the most important tools in biomedical and pharmaceutic research. The main advantage of this technique over the traditional bright field optical microscopy is the fact that it allows the selection of a thin cross-section of the sample by rejecting the visual information coming from the out-of-focus planes. However, the small amount of energy radiated by the fluorophore and the huge light amplification performed by the photon detector to capture this visual information introduces a type of multiplicative noise described by a Poisson distribution. Additionally, the radiation efficiency of the fluorophore decreases with the time, an effect called photobleaching, leading to a decrease in the image intensity along the time.

In this paper a reconstruction algorithm is proposed where the multiplicative noise and the photobleaching effect are modeled. The goal is to obtain the morphology and the intensity decay rate across the cell nucleus from a sequence of FCM images. The reconstruction algorithm is formulated as an optimization problem where a convex energy function is minimized. Tests using synthetic and real data are presented to illustrate the application of the algorithm and the effectiveness of the results.
\end{abstract}

Index Terms-Denoising, Poisson, Bayesian, confocal microscopy, convex optimization.

\section{INTRODUCTION}

The Fluorescence Confocal Microscopy (FCM) is nowadays one of the most important tools in biomedical and pharmaceutic research [1]. The main advantage of this technique over the traditional bright field optical microscopy is the fact that it allows the selection of a thin cross-section of the sample by rejecting the visual information coming from the out-of-focus planes (see Fig. 1). Additionally, it allows the use of fluorescence synthetic molecules, e.g. green fluorescent protein (GFP), that radiate in a wave length different from the one of the incident LASER. Using the right optical filters it is easy to track these molecules inside the cell, by observing only its emitted radiation. Tagging the protein of interest with GFP it is possible to follow these molecules aiming to understand the dynamic mechanisms behind the molecular machinery.

The most significant advances in this technique, occurred during the last decade, due to the improvement on the Laser Scanning Confocal Microscope (LSCM) [1], to the development of synthetic fluorescent probes and proteins and to the development of a wider spectrum of LASER light sources coupled to highly accurate acoustic/optic controlled filters.

Correspondent author: Isabel Rodrigues (irodrigues@isr.ist.utl.pt)

Partially supported by FCT, under ISR/IST plurianual funding (POSC program, FEDER)

Affiliation: ${ }^{1}$ Instituto Superior de Engenharia de Lisboa, ${ }^{2}$ Instituto de Sistemas e Robótica, ${ }^{3}$ Instituto Superior Técnico, Lisbon, Portugal

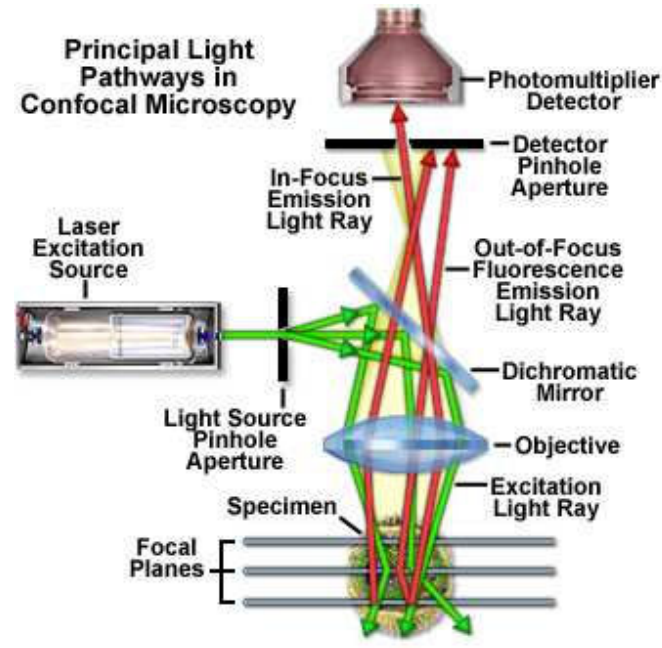

Fig. 1. Fluorescence Confocal Microscope (from Nikon-MicroscopyU),

The FCM images are in general very noisy, with small signal to noise ratio (SNR), corrupted by a type of multiplicative noise described by a Poisson distribution. Additionally, an intensity decreasing effect along the time, depending on the intensity of the incident LASER called photobleaching, is observed. The more intense is the incident LASER beam the larger is the intensity decay rate [2].

The Poisson noise arises in systems involving counting procedures such as PET/SPECT [3], functional MRI [4] and fluorescence confocal microscopy [2].

The photobleaching effect results from the GFP decreasing radiation emission efficiency. This occurs because the fluorophore permanently loses the ability to fluoresce, due to chemical reactions induced by the incident LASER or by other surrounding molecules. The decreasing on the image intensity is associated to a decreasing on the signal to noise ratio of the images, making the biological information recovery more and more difficult [5].

This photobleaching effect, however, enables the observation of the spatio-temporal dynamics of the fluorescently tagged proteins, namely with the use of GFP fusions expressed in living cells [6]. In fact, by strongly radiating a small region in nucleus, an abrupt photbleaching effect is induced in that region. The intensity in the rest of the nucleus will also decrease due to the random walk effect of the molecules that enter into the hole. Therefore, by observing the intensity decay rate in the cell nucleus it is possible to infer the diffusion velocity of these molecules. Some of them, however, are binded to large and almost static structures presenting very small diffusion rates. 
In this paper a denoising algorithm is formulated as an optimization problem where a convex energy function is minimized. Since the purpose is to achieve knowledge on the dynamics of the molecular process occurring along the time, the denoising methodology is performed in a time course basis instead of the traditional image basis. The convex formulation and the optimization procedure with the Newton algorithm guarantees a continuous convergence toward the global minimum in a small number of iterations. The algorithm assumes a constant cell morphology, $f_{i, j}$, with a pixel intensity decreasing along the time with a space varying decay rate, $\lambda_{i, j}$. The ultimate goal is to estimate these two fields from the sequence of images that were previously aligned using a simple correlation based method.

Tests with synthetic and real data are presented to illustrate the application and performance of the algorithm.

The paper is organized as follows. Section II formulates the problem, section III presents the experimental results using synthetic and real data and section IV concludes the paper.

\section{Problem Formulation}

The data used to reconstruct the cell nucleus is composed of a set of images acquired along the time. During the acquisition process the cell moves and rotates. Therefore, previous to reconstruction, an alignment procedure is needed to compensate for these displacements. In this work a simple correlation technique is used to align the images,

$$
T_{k, r}=\arg \max _{T} \operatorname{corr}\left(\mathbf{X}_{k}, T_{k, r}\left(\mathbf{X}_{r}\right)\right)
$$

where $\operatorname{corr}(\mathbf{X}, \mathbf{Y})$ is the correlation function between two images and $T_{k, r}$ is the rigid body transform that maximizes the correlation between the images $\mathbf{X}_{k}$ and $\mathbf{X}_{r}$. These transformations are computed for all pairs of consecutive images in the sequence, $T_{i, i+1}$, and the respective compensation performed. By stacking these aligned images, a volume data is obtained.

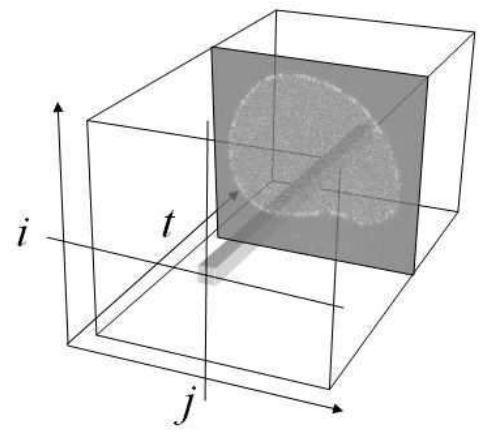

Fig. 2. Data tensor

Let $Y=\left\{y_{i, j}(t): 1 \leq i \leq N, 1 \leq j \leq M, 1 \leq t \leq T\right\}$ be the $3 D$ data tensor containing the collection of acquired $2 D$ images. Here, $t$ represents time and the ordered pairs $(i, j)$ denote pixel indexes within each image. Thus, the images are stacked along the temporal axis, as shown in Fig. 2. To each pixel $(i, j)$ corresponds a $T$-dimensional data vector $y_{i, j}:=\left[y_{i, j}(1) y_{i, j}(2) \cdots y_{i, j}(T)\right]$, obtained by collecting all the observations associated with the pixel $(i, j)$ along the $t$-axis. In this paper, the $T$-dimensional vectors $y_{i, j}$ are processed independently of one another. Thus, a total of $N \times M$ 1D signals must be processed but, since they are decoupled, our technique is amenable to a fast implementation in a parallel computing environment.

The noise corrupting the Fluorescent Confocal Microscopy (FCM) images is multiplicative and described by a Poisson distribution [7]. Furthermore, the time-decreasing intensity, called photobleaching [8], is modelled by a decaying exponential whose rate may vary from pixel to pixel (see Fig.4). To simplify notation, let $y=[y(1) y(2) \cdots y(T)]$ be the $T$-dimensional data vector associated with the pixel $(i, j)$ (previously denoted by $y_{i, j}$ ). In view of our assumptions, we have

$$
\begin{aligned}
g(t) & =f e^{-\lambda t} \\
p(y(t) \mid f, \lambda) & =\frac{(g(t))^{y(t)}}{y(t) !} e^{-g(t)}
\end{aligned}
$$

The goal is to estimate the deterministic parameters $f>0$ and $\lambda>0$ from the data vector $y$, by solving the associated Maximum Likelihoohd (ML) [9] optimization problem

$$
(\hat{f}, \hat{\lambda})=\arg \min _{f>0, \lambda>0} E(y, f, \lambda)
$$

where $E(y, f, \lambda)=-\log (p(y \mid f, \lambda))$.

Assuming statistical independence over time,

$$
p(y \mid \lambda, f)=\prod_{t=1}^{T} p(y(t) \mid f, \lambda)
$$

the ML problem boils down to

$$
(\hat{f}, \hat{\lambda})=\arg \min _{f>0, \lambda>0} \phi(f, \lambda)
$$

where

$$
\begin{aligned}
& \phi(f, \lambda)=c(\lambda) f-\alpha_{y} \log (f)+\beta_{y} \lambda \\
& c(\lambda):=\frac{1}{T} \sum_{t=1}^{T} e^{-\lambda t}=\frac{e^{-\lambda}}{T} \frac{1-e^{-\lambda T}}{1-e^{-\lambda}}
\end{aligned}
$$

with $\alpha_{y}:=(1 / T) \sum_{t=1}^{T} y(t)$ and $\beta_{y}:=(1 / T) \sum_{t=1}^{T}(t y(t))$.

Problem (6) is solved by first optimizing over $f$ since, for fixed $\lambda$, the function $\phi$ in (7) is convex with respect to $f$ and therefore the global minimizer corresponds to the stationary point $\hat{f}(\lambda)=\alpha_{y} / c(\lambda)$.

Plugging this back in (7) leaves the one-dimensional problem

$$
\hat{\lambda}=\arg \min _{\lambda>0} \psi(\lambda):=\alpha_{y} \log (c(\lambda))+\beta_{y} \lambda
$$

to be solved. It can be shown that the cost function $\psi$ in (9) is convex (proof omitted due to paper length constraints). Thus, Newton's algorithm

$$
\lambda_{k+1}=\lambda_{k}-s_{k} \dot{\psi}\left(\lambda_{k}\right) / \ddot{\psi}\left(\lambda_{k}\right)
$$

where $s_{k}$ denotes an Armijo step, will converge quadratically 
to the global minimizer from any initial point [10]. In fact, the following heuristic provides us with a good initial point: for $\lambda>0$ and large $T$, we have $e^{-\lambda T} \approx 0$, leading to the approximation $c(\lambda) \approx e^{-\lambda} /\left(T\left(1-e^{-\lambda}\right)\right.$, see (8). Inserting this in (9) yields

$$
\lambda^{*}=\arg \min _{\lambda>0}\left(\beta_{y}-\alpha_{y}\right) \lambda-\alpha_{y} \log \left(1-e^{-\lambda}\right)
$$

which can be solved in closed-form:

$$
\lambda^{*}=\log \left(\beta_{y} /\left(\beta_{y}-\alpha_{y}\right)\right)
$$

This point $\lambda^{*}$ can be used as an initialization for the Newton's algorithm.

The parameters $f$ and $\lambda$ were computed for the $1 \mathrm{D}$ vector associated to each pixel along the time, called time course, and two images $f_{i, j}$ and $\lambda_{i, j}$, with $0 \leq i, j \leq N, M$, were estimated and displayed

\section{EXPERIMENTAL RESULTS}

In this section the results obtained from tests using synthetic and real data are presented to illustrate the application of the algorithm.

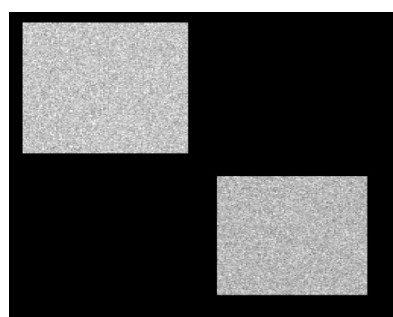

(a) $n=0$

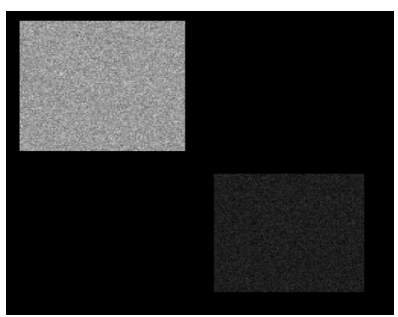

(c) $n=25$

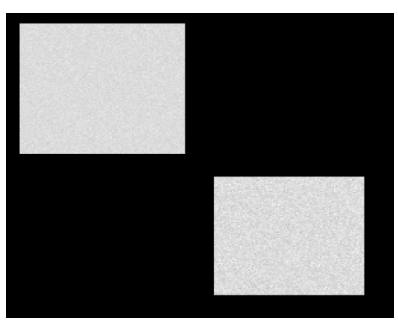

(e) $f_{i, j}$

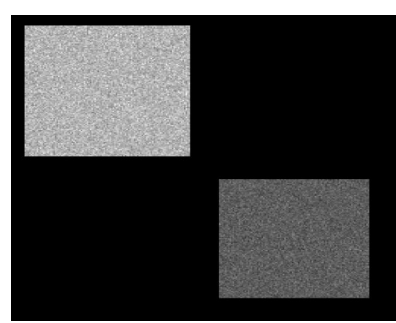

(b) $n=10$

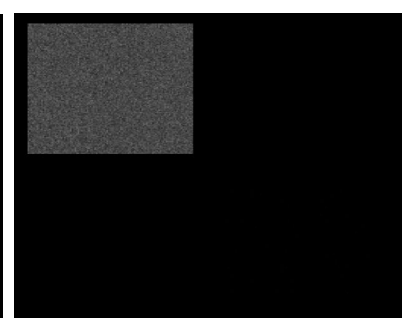

(d) $n=100$

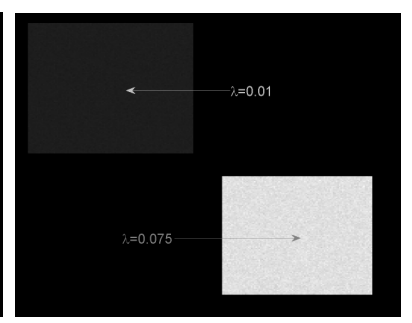

(f) $\lambda_{i, j}$
Fig. 3. Synthetic Data (a)-(d) and the resulting reconstructed images, $f_{i, j}$ (e) and $\lambda_{i, j}$ (f).

\section{A. Synthetic Data}

The synthetic data consists on a set of 100 images with $256 \times 256$ pixels. In the template image three different

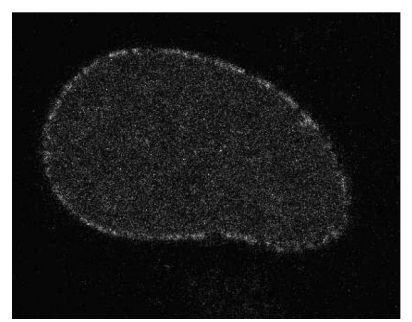

(a) $n=0$

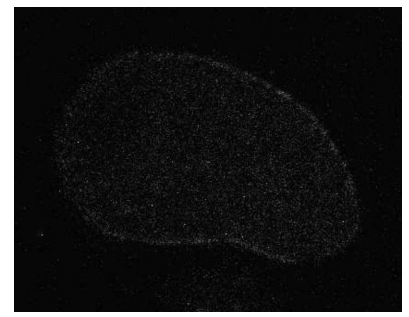

(c) $n=150$

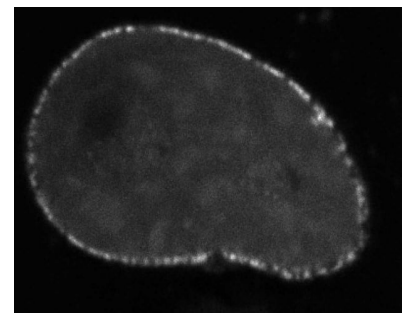

(e) $f_{i, j}$

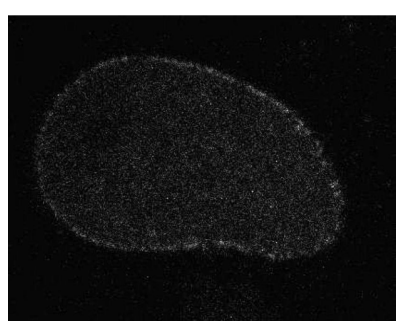

(b) $n=50$

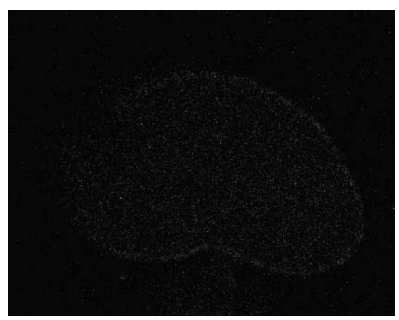

(d) $n=250$

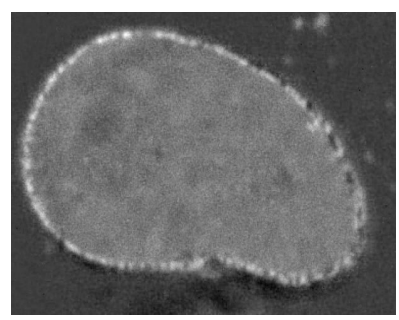

(f) $\lambda_{i, j}$

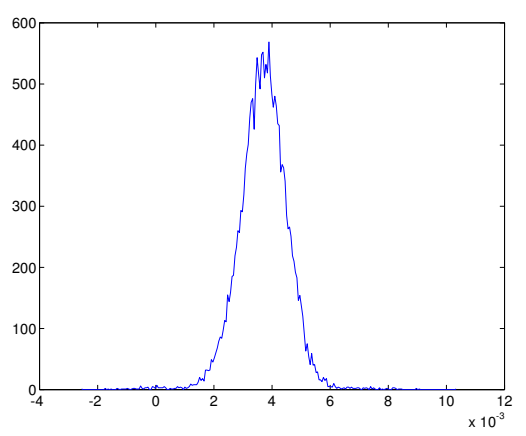

(g) $\lambda$ histogram inside the nucleus

Fig. 4. Real Data (a)-(d) and the resulting reconstructed images, $f_{i, j}$ (e) and $\lambda_{i, j}$ (f) and the respective histogram $(\mathrm{g})$.

regions were generated: two squares with intensity 200 and a zero intensity background. The intensity images were the result of applying exponential decays to the square regions with rates of $\lambda=0.01$ and $\lambda=0.075$ for the upper left square and the lower right square respectively and the 100 images were generated. The stack was then corrupted by Poisson noise (Fig.3 (a) - (d)). The methodology presented above was applied to these data and the results are displayed in Fig.3 (e) and (f). The mean values for $\lambda$ were computed in both squares: $\bar{\lambda}_{1}=0.01$ with a standard deviation of $\sigma_{1}=0.00045$ for the upper left square and $\bar{\lambda}_{2}=0.075$ and a standard deviation of $\sigma_{2}=0.0021$ for the lower right. 


\section{B. Real Data}

The real data is composed of a set of 273 images with $320 \times 420$ pixels obtained with a confocal LASER-scanning microscope (CLSM).

In confocal LASER-scanning microscopy a LASER beam is raster-scanned across a focal plane within the specimen as shown in Fig.5. The emitted light is processed, the out-offocus information rejected by a pinhole and the remaining light directed to a detector to produce a digital image in a computer. In this technique it is possible to define a small region in the specimen where the incident LASER is more intense and so is the photobleaching; in the image it appears as a darker region. In fact, this region is the main source of the photobleaching effect observed in the whole image. The green fluorescent protein (GFP) molecules that are observed in this type of images diffuse across the image entering and leaving the hot spot region according to a random walk process. However, inside the hole, these proteins loose their fluorescence properties and when they get out to the rest of the cell they contribute less to the mean intensity of the image, leading to a substantial decrease on the intensity of the observed image along the time.

In Fig.4 (a)-(d), four of the 273 data images that constitute the sequence are shown. Fig.4 (e) and (f) present images of the estimated parameters $f_{i, j}$ (e) and $\lambda_{i, j}$ (f). In these images it is possible to observe the hot spot (the black hole) where a high intensity LASER beam is focused. The decay rate is approximately constant across the image, inside the nucleus. Fig.4 (g) shows the histogram of $\lambda_{i, j}$ inside the nucleus which assumes values mainly in the interval $[0.002,0.005]$.

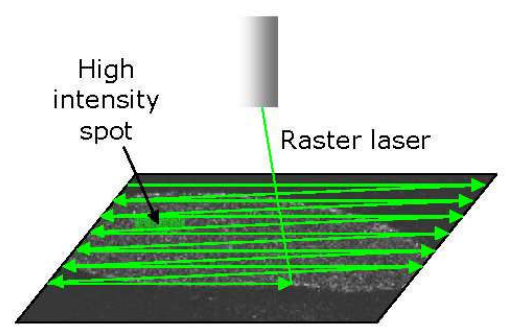

Fig. 5. Confocal Laser-Scanning Microscope (CLSM).

\section{CONCLUSions}

In this paper a reconstruction algorithm is proposed to fluorescent confocal LASER scanning microscopy imaging. In this modality the images are corrupted by a type of multiplicative noise described by a Poisson distribution, due to the huge light amplification performed by the photon detectors, in order to make the light emitted by the GFP visible. Additionally, the emission efficiency of these proteins decreases along the time leading to a decreasing effect on the image intensity called photobleaching. In this paper a statistical algorithm is proposed to estimate the morphology of the cell nucleus as well as the decay rate for each location. The estimation of this decay rate is important to compensate for the photobleaching effect but also because it may contain relevant biological information, namely, protein flow information across the nucleus.

The reconstruction algorithm processes the data in a time course basis, that is, the data associated with each pixel along the time is processed independently of the other pixels.

The estimation algorithm is based on the minimization of an energy function with respect to the $f_{i, j}$ and $\lambda_{i, j}$ parameters. In the context of the presented methodology, the optimization problem is convex which guarantees the convergence to the global minimum and therefore to the optimum solution.

Synthetic and real data are used to illustrate the application of the algorithm. The synthetic example shows the ability of the algorithm to find the true solution.

\section{ACKNOWLEDGMENT}

The authors thank Dr. José Rino and $\operatorname{Prof}^{a}$ Maria do Carmo Fonseca, from the Instituto de Medicina Molecular de Lisboa, for providing biological technical support and the data used in this paper.

\section{REFERENCES}

[1] J. B. Pawley, The Handbook of Biological Confocal Microscopy. New York: Plenum Press, 1995.

[2] G. van Kempen, L. van Vliet, and P. Verveer, "Application of image restoration methods for confocal fluorescence microscopy," in Proc. SPIE, 3-D Microscopy: Image Acquisition and Processing IV, T. W. C.J. Cogswell, J.-A. Conchello, Ed., vol. 2984. SPIE, 1997, pp. $114-124$.

[3] J. M. Ollinger and J. A. Fessler, "Positron emission tomography," IEEE Signal Processing Magazine, vol. 14, no. 1, pp. 43-55, Jan. 1997.

[4] G. E. Hagberg, G. Zito, F. Patria, and J. N. Sanes, "Improved detection of event-related functional mri signals using probability functions," NeuroImage, vol. 2, pp. 1193-1205, 2001.

[5] N. A. M. A. Lashin, "Restoration methods for biomedical images in confocal microscopy," Ph.D. dissertation, Berlin, 2005.

[6] K. Braeckmans, "Fotobleaching with the confocal laser scanning micrscope for mobility measurments and the encoding of microbeads," Ph.D. dissertation, University of Gent, 2004.

[7] P. J. Verveer, M. J. Gemkow, and T. M. Jovin, "A comparison of image restoration approaches applied to three-dimensional confocal and wide-field fluorescence microscopy." J Microsc, vol. 193, no. 1, pp. 50-61, 1999.

[8] R. Underwood, "Frap and flip, photobleaching technique to reveal cell dynamics," 2007.

[9] T. K. Moon and W. C. Stirling, Mathematical methods and algorithms for signal processing, 2000.

[10] S. Boyd and L. Vandenberghe, Convex Optimization. Cambridge University Press, March 2004. 